\title{
Association of time-to-treatment with outcomes of Pneumocystis pneumonia with respiratory failure in HIV-negative patients
}

\author{
Ryoung-Eun Ko ${ }^{1}$, Soo Jin Na', Kyungmin Huh', Gee Young Suh ${ }^{1,3}$ and Kyeongman Jeon ${ }^{1,3^{*}}$ (D)
}

\begin{abstract}
Background: The prevalence of pneumocystis pneumonia (PCP) and associated hypoxic respiratory failure is increasing in human immunodeficiency virus (HIV)-negative patients. However, no prior studies have evaluated the effect of early anti-PCP treatment on clinical outcomes in HIV-negative patient with severe PCP. Therefore, this study investigated the association between the time to anti-PCP treatment and the clinical outcomes in HIV-negative patients with PCP who presented with hypoxemic respiratory failure.
\end{abstract}

Methods: A retrospective observational study was performed involving $51 \mathrm{HIV}$-negative patients with PCP who presented in respiratory failure and were admitted to the intensive care unit between October 2005 and July 2018. A logistic regression model was used to adjust for potential confounding factors in the association between the time to anti-PCP treatment and in-hospital mortality.

Results: All patients were treated with appropriate anti-PCP treatment, primarily involving trimethoprim/ sulfamethoxazole. The median time to anti-PCP treatment was 58.0 (28.0-97.8) hours. Thirty-one (60.8\%) patients were treated empirically prior to confirmation of the microbiological diagnosis. However, the hospital mortality rates were not associated with increasing quartiles of time until anti-PCP treatment ( $P=0.818$, test for trend). In addition, hospital mortality of patients received early empiric treatment was not better than those of patients received definitive treatment after microbiologic diagnosis $(48.4 \%$ vs. $40.0 \%, P=0.765)$. In a multiple logistic regression model, the time to anti-PCP treatment was not associated with increased mortality. However, age (adjusted OR 1.07, 95\% Cl 1.01-1.14) and failure to initial treatment (adjusted OR 13.03, 95\% Cl 2.34-72.65) were independently associated with increased mortality.

Conclusions: There was no association between the time to anti-PCP treatment and treatment outcomes in HIVnegative patients with PCP who presented in hypoxemic respiratory failure.

Keywords: Pneumocystis pneumonia, HIV seronegativity, Respiratory insufficiency, Time-to-treatment, Treatment outcome

\section{Background}

Pneumocystis pneumonia (PCP), a pulmonary infection caused by Pneumocystis jirovecii, remains one of the most prevalent opportunistic infections in immunocompromised

\footnotetext{
*Correspondence: kjeon@skku.edu

'Department of Critical Care Medicine, Samsung Medical Center, Sungkyunkwan University School of Medicine, 81 Irwon-ro, Gangnam-gu, Seoul 06351, Republic of Korea

${ }^{3}$ Division of Pulmonary and Critical Care Medicine, Department of Medicine, Samsung Medical Center, Sungkyunkwan University School of Medicine, 81 Irwon-ro, Gangnam-gu, Seoul 06351, Republic of Korea

Full list of author information is available at the end of the article
}

patients [1-3]. The development of highly active antiretroviral therapy and effective prophylaxis against PCP have reduced the mortality of PCP in patients with human immunodeficiency virus (HIV) $[4,5]$. However, as the numbers of patients receiving immunosuppressive therapy after organ transplantation and antitumor chemotherapy increase, the incidence of PCP in HIV-negative patients has also increased [2, 6-8]. Typically, HIV-positive patients follow a more insidious course [2, 9]. In contrast, HIVnegative patients with PCP present with abrupt-onset hypoxemic respiratory failure $[9,10]$. Therefore, it is necessary

(C) The Author(s). 2019 Open Access This article is distributed under the terms of the Creative Commons Attribution 4.0 International License (http://creativecommons.org/licenses/by/4.0/), which permits unrestricted use, distribution, and 
to treat the infection before complications from respiratory failure arise. However, early treatment is sometimes difficult, as the diagnosis of PCP is mostly a clinical one in critical care settings [9].

Over the past several decades, trimethoprim/sulfamethoxazole (TMP/SMX) remains the drug of choice for PCP $[2,9,11]$. Because of the high mortality rate in HIV-negative patients, it has been suggested that antiPCP treatment should be started empirically in patients who are suspected to have moderate to severe PCP [12]. However, TMP/SMX induces several side effects, including granulocytopenia, skin eruptions, hepatotoxicity, and renal toxicity. Using TMP/SMX inappropriately can also allow resistant strains to develop [13]. There is a lot of literature regarding PCP in HIV-negative patients; however, previous studies have focused on patient factors and laboratory findings associated with clinical outcomes $[14,15]$. Few have investigated the delayed initiation of anti-PCP treatment therapy in HIV-negative patients compared to HIV-positive patients [16, 17]. No prior study has evaluated the effect of early anti-PCP treatment on the clinical outcomes in HIV-negative patients with severe PCP.

Therefore, this study investigated the association between the time to anti-PCP treatment and the clinical outcomes in HIV-negative patients with PCP who presented with hypoxemic respiratory failure.

\section{Methods}

We retrospectively reviewed the medical records of all consecutive patients with $\mathrm{PCP}$ who were admitted to the medical intensive care unit (ICU) for respiratory failure at Samsung Medical Center (a 1979-bed, university-affiliated, tertiary referral hospital in Seoul, South Korea) between October 2005 and July 2018. The study was approved by the Institutional Review Board of Samsung Medical Center. Informed consent was waived because of the retrospective observational nature of the study. All patient records and data were anonymized and de-identified prior to analysis.

\section{Study population}

All consecutive patients older than 20 years who were admitted to the medical ICU through the emergency department (ED) for acute respiratory failure were screened for inclusion (Fig. 1). Some of the clinical data from patients who were enrolled between 2005 and 2011 were also included in the previous study [18]. Patients were included if they had a microbiologically confirmed diagnosis of PCP and required respiratory support with mechanical ventilation including non-invasive ventilation or high-flow nasal cannula for respiratory failure. Patients were excluded if they had a positive HIV antibody test. Finally, patients who had neither respiratory symptom nor abnormal

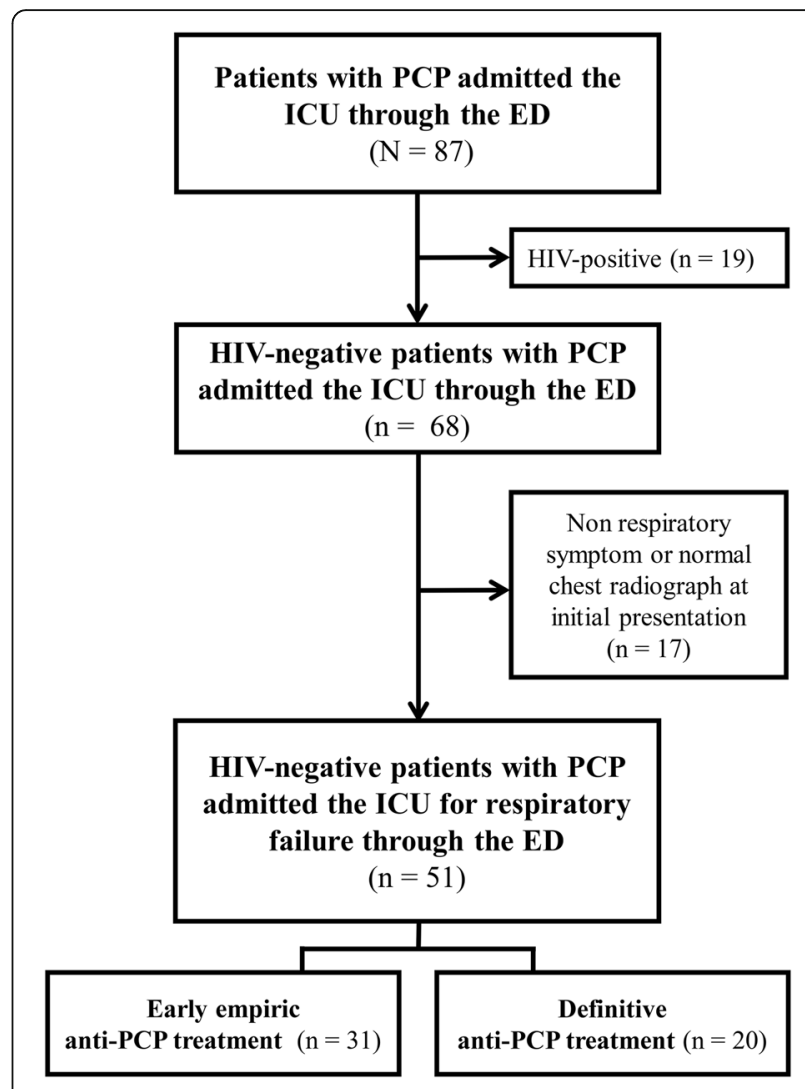

Fig. 1 Study flow diagram. PCP, Pneumocystis pneumonia; ICU, intensive care unit; ED, emergency department

finding on chest radiographs at initial presentation were excluded from the study. In patients who had multiple admissions for acute respiratory failure due to $\mathrm{PCP}$ during the study period, only the first ICU admission was evaluated.

The diagnosis of PCP was based on clinical symptoms and the presence of a new infiltration on chest radiograph, along with morphological identification of the organism in bronchoalveolar lavage (BAL) fluid or lung tissue obtained by transbronchial lung biopsy (TBLB). The BAL fluid samples were stained using Gram and Ziehl-Neelsen methods and then cultured for bacteria, mycobacteria, and fungi. Multiplex nested polymerase chain reaction (PCR) assays were used to detect the following: influenza viruses $A$ and $B$; parainfluenza viruses 1,2 , and 3; respiratory syncytial virus; and adenovirus [19]. Quantitative real-time PCR was used to measure the cytomegalovirus (CMV) deoxyribonucleic acid (DNA) in the BAL fluid [20]. Microbiological identification of P.jirovecii was confirmed by documenting the organism with Wright Giemsa or Gram-Weigert stain, or the cyst with the Gomori methenamine silver or calcofluor white stain [2]. 


\section{Data collection}

The following data were extracted from the medical records: general demographic information, underlying diseases, medications used during the previous month, PCP prophylaxis, need for renal replacement therapy, need for vasopressor support, anti-PCP medication, and newly developed organ failure during the ICU stay. We also collected the following laboratory data from the medical records: the white blood cell count, absolute neutrophil count, absolute lymphocyte count, albumin, C-reactive protein, arterial partial pressure of oxygen $\left(\mathrm{PaO}_{2}\right) /$ fraction of inspired oxygen $\left(\mathrm{FiO}_{2}\right)$ ratio ( $\mathrm{PF}$ ratio), and alveolar-arterial oxygen gradient $\left[\mathrm{D}(\mathrm{A}-\mathrm{a}) \mathrm{O}_{2}\right]$. The severity of illness was assessed using the Simplified Acute Physiology Score 3 (SAPS 3) and Sequential Organ Failure Assessment (SOFA) score [21, 22]. Finally, we studied the clinical outcomes, including the length of ICU stay, the length of hospital stay, and ICU and in-hospital mortality.

The time to anti-PCP treatment interval was defined as the number of hours from the time of emergency room arrival to the initial administration of antiPneumocystis antibiotics. We classified patients into two groups according to the timing of initial anti-PCP treatment before or after microbiologic diagnosis [23]: early empiric treatment group and definitive treatment group. The doses of corticosteroids used for immunosuppression and the adjunctive therapy for PCP were expressed as the prednisolone-equivalent doses [24]. The adjunctive corticosteroid therapy was defined as that started within $72 \mathrm{~h}$ of initiating the specific anti-PCP treatment (consisting of at least $40 \mathrm{mg}$ prednisone twice daily for 5 days regardless of the subsequent tapering schedule), and the use of corticosteroids before the onset of PCP [25]. The failure of anti-PCP treatment was defined as follows: (1) progressive clinical deterioration as demonstrated by the inability to maintain a stable $\mathrm{PaO}_{2}$ despite an increase in $\mathrm{FiO}_{2}$; and (2) progressive deterioration of vital signs with an increased $\mathrm{FiO}_{2}$ requirement despite 7 days of appropriate therapy $[11,26]$. Breakthrough PCP infection was defined as that diagnosed in a patient receiving prophylactic agents with known activity against $P$. jirovecii for at least 7 days prior to the diagnosis [27].

\section{Statistical analysis}

All data are presented as medians and interquartile range (IQR) for continuous variables, and as numbers and percentages for categorical variables. Continuous variables were analyzed using the Mann-Whitney-Wilcoxon U test. Categorical variables were analyzed using the Pearson $X^{2}$ test or Fisher exact test. The Mantel-Haentzel trend test was used to examine the trends in the hospital mortality rate across the time quartiles to anti-PCP treatment [28]. To adjust for potential confouding factors in the association between the time to anti-PCP treatment and in-hospital mortality, multiple logistic regression analysis was used. Finally, we compared baseline characteristics and clinical outcomes between early empiric treatment group and definitive treatment group. Probabilities of survival after anti-PCP treatment for each group were estimated by Kaplan-Meier method and compared by the logrank test. Variables with a $P$-value less than 0.2 on univariate analyses [29], as well as a priori variables of age and sex were entered into a multiple logistic regression model in which in-hospital mortality was the outcome variable of interest. To reduce the risk of multicollinearity, one closely correlated variable was a candidate for inclusion in the final model. Data are presented as adjusted odds ratios (OR) with 95\% confidence intervals (CI). All of the tests were two-sided. $P$ values $<0.05$ were considered statistically significant. All analyses were performed using SPSS for Windows (ver. 23.0; IBM Corp., Armonk, NY, USA).

\section{Results \\ Study population}

During the study period, a total of 117 patients with PCP were admitted to the ICU for respiratory support. Nineteen of these patients were excluded from the study because they had HIV infections. After excluding patients who were initially admitted to general ward from outpatient care with other cause $(n=29)$, those who were transfer to our hospital $(n=1)$, and those with an initial chief complaint that was unrelated to respiratory symptoms $(n=17), 51$ patients with acute hypoxemic respiratory failure and a new infiltration on chest radiograph were included.

The patients' baseline characteristics are summarized in Table 1. The median patient age was 52.0 (IQR 40.5$66.0)$ years. Thirty-five (68.6\%) patients were men. The main underlying conditions associated with the development of PCP included malignancies $(n=28,54.9 \%)$ and history of solid organ transplant $(n=18,35.3 \%)$. All of the patients were immunosuppressed before developing PCP. Twenty-two (43.1\%) patients received systemic steroids. The median prednisolone-equivalent dose was $16.1(6.2-34.7) \mathrm{mg} /$ day in patients who received systemic steroids. Two (3.9\%) patients had a history of receiving prophylactic TMP/SMZ.

At the time of ICU admission, all of the patients had hypoxemia requiring mechanical ventilation $(n=43$, $84.3 \%)$ or high-flow nasal cannula support $(n=8,15.7 \%)$. The median PF ratio was $137.2(112.8-164.6) \mathrm{mmHg}$. The $\mathrm{D}(\mathrm{A}-\mathrm{a}) \mathrm{O}_{2}$ was 39.9 (30.8-52.3) mmHg. Twelve (23.5\%) patients required vasopressor support and one (2.0\%) needed renal replacement therapy. The median SAPS 3 and SOFA scores on ICU admission were 48.0 (37.0-57.5) and 6.0 (5.0-8.0), respectively. 
Table 1 Baseline patient characteristics

\begin{tabular}{|c|c|}
\hline Characteristics & No. of patients (\%) or median (IQR) \\
\hline Age, years & $52.0(40.5-66.0)$ \\
\hline Sex, male & $35(68.6)$ \\
\hline \multicolumn{2}{|l|}{ Underlying disease } \\
\hline Malignancy & $28(54.9)$ \\
\hline Hematologic & 21 \\
\hline Solid & 7 \\
\hline Solid organ transplant & $18(35.3)$ \\
\hline Kidney & 11 \\
\hline Liver & 4 \\
\hline Heart & 2 \\
\hline Lung & 1 \\
\hline Other $^{a}$ & $5(9.8)$ \\
\hline \multicolumn{2}{|l|}{ Immunosuppressive agents used, previous month ${ }^{\mathrm{b}}$} \\
\hline Steroid only & $18(35.3)$ \\
\hline Chemotherapy only & $29(56.9)$ \\
\hline Steroid with chemotherapy & $4(7.8)$ \\
\hline Prednisolone-equivalent dose, mg, if steroid used & $16.1(6.2-34.7)$ \\
\hline Pneumocystis prophylaxis & $2(3.9)$ \\
\hline \multicolumn{2}{|l|}{ Chest radiography findings } \\
\hline Pleural effusion & $11(21.6)$ \\
\hline \multicolumn{2}{|l|}{ Radiographic pulmonary pattern } \\
\hline Focal or diffuse alveolar pattern & $24(47.1)$ \\
\hline Focal or diffuse interstitial pattern & $5(9.8)$ \\
\hline Focal or diffuse alveolar-interstitial pattern & $22(43.1)$ \\
\hline \multicolumn{2}{|l|}{ Laboratory findings on ICU admission } \\
\hline WBC blood cells,/ $\mu \mathrm{L}$ & $7960.0(5320.0-12,225.0)$ \\
\hline 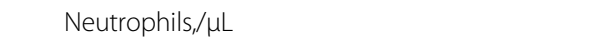 & $5458.0(3245.0-10,395.0)$ \\
\hline Lymphocytes,/ML & $580.0(324.0-1020.0)$ \\
\hline Albumin, $\mathrm{g} / \mathrm{dL}$ & $2.8(2.4-3.3)$ \\
\hline $\mathrm{CRP}, \mathrm{mg} / \mathrm{dL}$ & $12.4(8.2-19.6)$ \\
\hline $\mathrm{PaO}_{2} / \mathrm{FiO}_{2}$ ratio, $\mathrm{mmHg}$ & $137.2(112.8-164.6)$ \\
\hline $\mathrm{D}(\mathrm{A}-\mathrm{a}) \mathrm{O}_{2}, \mathrm{mmHg}$ & $39.9(30.8-52.3)$ \\
\hline \multicolumn{2}{|l|}{ Organ failure } \\
\hline Shock & $12(23.5)$ \\
\hline Renal failure requiring renal replacement therapy & $1(2.0)$ \\
\hline \multicolumn{2}{|l|}{ Respiratory support on ICU admission day } \\
\hline Mechanical ventilation & $43(84.3)$ \\
\hline High-flow nasal cannula & $8(15.7)$ \\
\hline \multicolumn{2}{|l|}{ Severity of illness } \\
\hline SAPS 3 & $48.0(37.0-57.5)$ \\
\hline SOFA & $6.0(5.0-8.0)$ \\
\hline
\end{tabular}

No. number, IQR interquartile range, ICU intensive care unit, $W B C$ white blood cell, $C R P$ C-reactive protein, $\mathrm{PaO}_{2} / \mathrm{FiO}_{2}$ arterial partial pressure of oxygen/fraction of inspired oxygen, $D(A-a) O_{2}$ alveolar-arterial oxygen gradient, SAPS 3 Simplified Acute Physiology Score 3, SOFA Sequential Organ Failure Assessment

${ }^{a}$ Others include 2 glomerulonephritis, 2 interstitial lung disease, and 1 liver cirrhosis

${ }^{\mathrm{b}}$ Chemotherapy includes 4 patients with T-cell immunosuppressant 


\section{Diagnosis and treatment of PCP}

All of the patients underwent bronchoscopy with BAL \pm TBLB within 57.7 (30.2-91.7) hours of the ER visit (Table 2). The diagnosis of PCP was based on microbiological identification of $P$. jirovecii in the BAL fluid $(n=$ $44,86.3 \%)$ or lung biopsy specimens ( $n=14,27.5 \%)$. Seven (13.7\%) patients were positive for $P$. jirovecii in both the BAL fluid and pathology specimens. In addition to $P$. jirovecii, other pathogens were simultaneously isolated from respiratory specimens at the time of diagnosis. The most common simultaneously isolated pathogen was CMV in 13 patients (25.5\%), bacteria in 12 (23.5\%), and viruses other than CMV in 7 (13.7\%).

All of the patients were treated with appropriate antiPCP treatment, primarily involving TMP $(20 \mathrm{mg} / \mathrm{kg} /$ day)/SMZ (100 mg/kg/day). Thirty-one (60.8\%) patients were treated with TMP/SMZ empirically prior to confirmation of the microbiological diagnosis. The median time to anti-PCP treatment was 58.0 (28.0-97.8) hours. Baseline characteristics and the hospital mortality rates according to the time to anti-PCP treatment in quartiles are presented in Additional file 1: Table S1 and Fig. 2, respectively. Interestingly, the hospital mortality rates were not associated with increasing quartiles of time to anti-PCP treatment $(P=0.818$, test for trend).

All of the patients except one $(98.0 \%)$ were treated with adjunctive corticosteroids. One patient had an adverse reaction to TMP/SMZ involving an electrolyte imbalance after 7 days of use. After excluding this patient, the initial treatment response was assessed on day 7. Of the 50 patients, $20(40.0 \%)$ did not respond to the initial treatment with TMP/SMZ. These 20 patients were subsequently treated with clindamycin-primaquine $(n=14)$ or pentamidine $(n=6)$ as salvage therapy for PCP.

During their ICU stays, organ failure newly developed in $33(64.7 \%)$ patients, which included shock requiring vasopressors in 30 patients and acute kidney injury requiring renal replacement therapy in 13 (Table 3). The ICU mortality was $37.3 \%$ with a median stay of 15.0 (6.0-29.0) days. Ultimately, $23(45.1 \%)$ patients died while hospitalized. The median hospital stay was 24.0 (15.5-32.5) days.

The early empiric treatment group contained 31 (60.8\%) patients and the definitive treatment group contained 20 (39.2\%) patients. There were no significant differences in baseline characteristics, and diagnosis and treatment of PCP, except of the median time to antiPCP treatment ( $34.2 \mathrm{~h}$ for early empiric treatment group vs. $91.7 \mathrm{~h}$ for definitive treatment group, $P<0.001$ ) (Additional file 2: Table S2 and Additional file 3: Table S3). In comparison of clinical outcomes between the two groups, there was no significant difference in mortalities and length of stay (Table 3). In addition, early empiric treatment did not significantly affect overall survival (Fig. 3). Although the probability of survival for early empiric treatment group appears to increase in the first few weeks after anti-PCP treatment, this difference is not statistically significant $(P=0.8673$, log-rank test).

Table 2 Diagnosis and treatment of Pneumocystis pneumonia and clinical outcomes

\begin{tabular}{ll}
\hline Variables & No. of patients (\%) or median (IQR) \\
\hline Microbiological diagnosis & $39(76.5)$ \\
Bronchoalveolar lavage fluid & $12(23.5)$ \\
Lung biopsy specimen ${ }^{\text {a }}$ & $58.0(28.0-97.8)$ \\
Time to anti-PCP treatment, hours & $31(60.8)$ \\
Empiric treatment & $13(25.5)$ \\
Other pathogens identified from respiratory specimens & $7(13.7)$ \\
Cytomegalovirus & $11(21.6)$ \\
Virus other than cytomegalovirus ${ }^{b}$ & 5 \\
Bacteria & 4 \\
$\quad$ MRSA & 4 \\
$\quad$ Acinetobacter & 2 \\
$\quad$ Pseudomonas & $22(43.1)$ \\
Initial treatment regimen & \\
Trimethoprim/sulfamethoxazole & $51(100.0)$ \\
Adjunctive corticosteroid treatment & 50 (98.0) \\
\hline
\end{tabular}

No. number, IQR interquartile range, $P C P$ Pneumocystis pneumonia, MRSA methicillin-resistant Staphylococcus aureus

a 7 patients had positive results for the presence of $P$.jirovecii in both BAL fluid and biopsy specimens

biruses other than cytomegalovirus included rhinovirus $(n=3)$, coronavirus $(n=2)$, and rhinovirus $(n=2)$ 


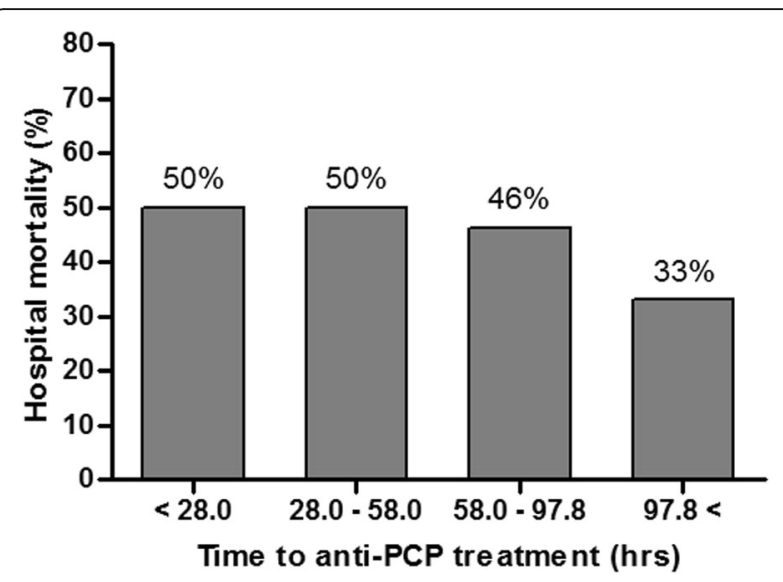

Fig. 2 Trends in hospital mortality rate according to time to antiPCP treatment in quartiles. PCP, Pneumocystis pneumonia

\section{Factors associated with increased hospital mortality}

Univariate comparisons of the clinical characteristics of hospital survivors and non-survivors are presented in Table 4. Compared to survivors, non-survivors were likely to be older (48.0 [34.5-59.0] vs. 64.0 [50.5-69.0], $P=0.007)$, require more mechanical ventilation $(67.9 \%$ vs. $100 \%, P=0.009)$, SAPS 3 score increase (43.0 [30.5-54.5] vs. 56.0 [46.5-58.0], $P=$ $0.030)$, more CMV co-infection $(10.7 \%$ vs. $43.5 \%, P=$ $0.011)$, and failed to initial anti-PCP treatment $(21.4 \%$ vs. $69.6 \%, P=0.002)$. After adjusting for potential confounding factors, age (adjusted OR 1.07, 95\% CI 1.01-1.14, $P=0.010$ ) and failure to initial treatment (adjusted OR 13.03, 95\% CI 2.34-72.65, $P=0.005$ ) were independently associated with increased mortality (Table 5). However, the time to anti-PCP treatment was not associated with increased mortality.

\section{Discussion}

This study investigated the association between the time to anti-PCP treatment and clinical outcomes in HIVnegative patients with PCP who presented with hypoxemic respiratory failure. Our results suggest that the clinical outcomes in HIV-negative patients with PCP and respiratory failure are unrelated to the time to initiation of anti-PCP treatment. In addition, clinical outcomes of patients received early empiric treatment were not better than those of patients received definitive treatment after microbiologic diagnosis.

Over the last decade, there has been a substantial decline in PCP-related mortality among HIV-positive patients, while there is increasing mortality (from PCP) in HIV-negative patients $[9,10]$. Delay in therapy in HIVnegative patients compared to HIV-positive patients was associated with higher mortality $[16,17]$. This finding suggests that empiric therapy for PCP should be initiated in patients with high clinical suspicion for PCP. Unfortunately, there is no clinical tool that rapidly identifies patients at risk of PCP in whom empiric treatment is warranted. In addition, the prevalence of PCP in HIVnegative cancer patients with acute respiratory failure and diffuse pulmonary infiltrates is $<10 \%[30,31]$. Empiric therapy for PCP in most patients, therefore, would be unnecessary and potentially cause harm.

Although several prior studies found that delayed therapy in anti-PCP treatment was associated with higher mortality [16, 17], we found no such difference. It is difficult to explain this discrepancy. It may be due to the difference in the units that were used to measure delayed treatment (hours vs. days). Unlike in previous studies, we measured the time to anti-PCP treatment was calculated in hours instead of days [32]. Another potential explanation for these conflicting results is

Table 3 Clinical ICU course

\begin{tabular}{|c|c|c|c|c|}
\hline Characteristics & $\begin{array}{l}\text { Overall } \\
(N=51)\end{array}$ & $\begin{array}{l}\text { Early empiric treatment } \\
(n=31)\end{array}$ & $\begin{array}{l}\text { Definitive treatment } \\
(n=20)\end{array}$ & $P$ value \\
\hline \multicolumn{5}{|l|}{ New development of organ failure ${ }^{a}$} \\
\hline Shock & $30(58.8)$ & $20(64.5)$ & $10(50.0)$ & 0.461 \\
\hline Renal failure requiring renal replacement therapy & $13(25.5)$ & $6(19.4)$ & $7(35.0)$ & 0.356 \\
\hline Extracorporeal membrane oxygenation & $2(3.9)$ & $2(6.5)$ & $0(0.0)$ & 0.674 \\
\hline \multicolumn{5}{|l|}{ Outcomes } \\
\hline ICU mortality & $19(37.3)$ & $13(41.9)$ & $6(30.0)$ & 0.573 \\
\hline Length of stay in ICU (days) & $15.0(6.0-29.0)$ & $18.0(8.5-30.5)$ & $11.0(5.0-19.0)$ & 0.162 \\
\hline Hospital mortality & $23(45.1)$ & $15(48.4)$ & $8(40.0)$ & 0.765 \\
\hline Length of stay in hospital (days) & $24.0(15.5-32.5)$ & $25.0(16.0-34.5)$ & $20.5(15.5-32.0)$ & 0.493 \\
\hline
\end{tabular}

Data are presented as number (percentage) or as median (interquartile range)

ICU intensive care units

${ }^{a}$ Two patients developed shock and renal failure requiring both renal replacement therapy and extracorporeal membrane oxygenation support. Eight patients developed both shock and renal failure requiring renal replacement therapy 


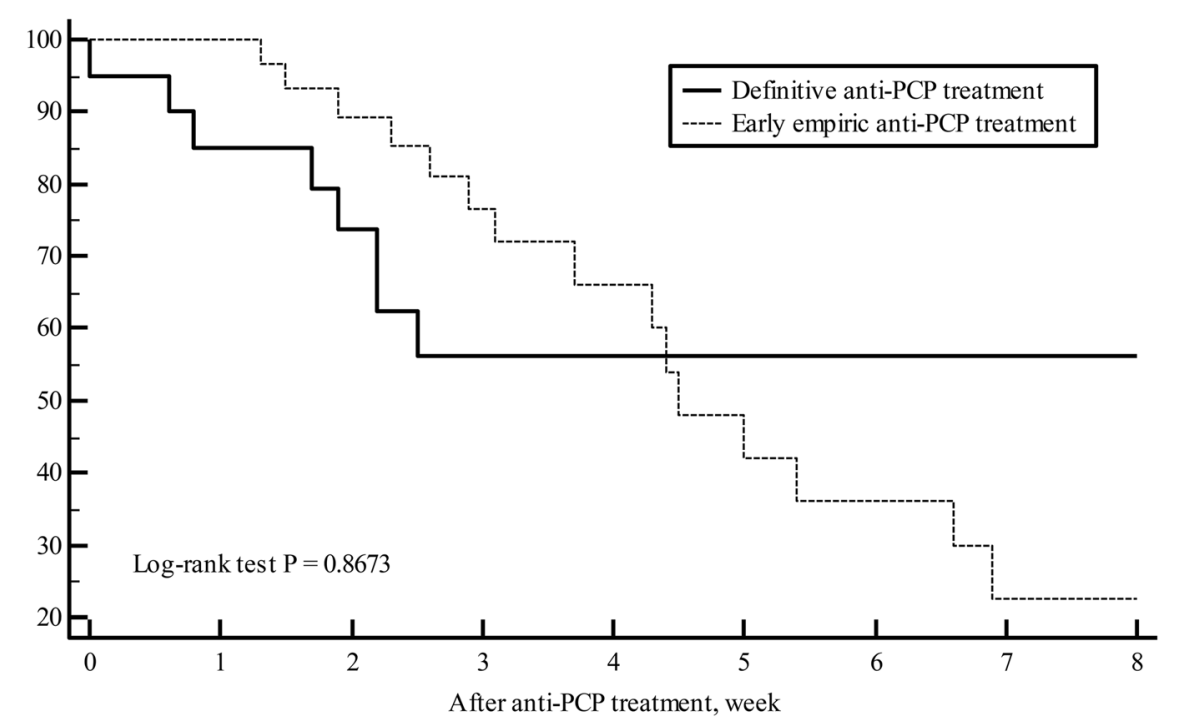

Number at risk

Group: Definitive anti-PCP treatment

\begin{tabular}{|c|c|c|c|c|c|c|c|}
\hline \multirow{2}{*}{\multicolumn{8}{|c|}{$\begin{array}{ccc}19 & 16 & 13 \\
\text { Group: Early empiric anti-PCP treatment }\end{array}$}} \\
\hline & & & & & & & \\
\hline 31 & 30 & 23 & 17 & 11 & 7 & 6 & 3 \\
\hline
\end{tabular}

Fig. 3 Kaplan-Meier curves of the probability of survival in patients who received early empiric anti-Pneumocystis pneumonia (PCP) treatment before microbiologic diagnosis (early empiric treatment group; dotted line) and those who received definitive anti-PCP treatment after microbiologic diagnosis (definitive treatment group; solid line)

differences in the study populations. We only included HIV-negative patients in our analysis of the association of time to anti-PCP treatment on mortality. In addition, the majority of patients required mechanical ventilation on admission. These results suggest against empiric treatment PCP in HIV-negative patients who present with hypoxemic respiratory failure given the various (potentially harmful) side effects of this treatment.

PCP in HIV-negative patients causes acute fulminant pneumonia with abrupt-onset respiratory failure and the need for mechanical ventilation $[9,10]$. We observed a high mortality rate in our patients who required mechanical ventilation, which was consistent with the findings from previous reports [14-16, 18]. In addition, the severity of illness (at the time of ICU admission) was associated with higher mortality. These findings suggest that a patient's general condition and underlying disease associated with PCP are more important than is early initiation of anti-PCP treatment in HIV-negative patients.

Given its high efficacy and bioavailavility, TMP/SMZ is used as a first-line agent in the treatment of PCP in patients with or without HIV infection [2, 9, 11]. Despite this, treatment failure has been reported with TMP/ SMZ in $10-40 \%$ of patients [2]. Although large randomized trials have shown the efficacy of TMP/SMZ in HIVpositive patients, only a few small observational studies have demonstrated this in HIV-negative patients $[9,10]$.
Therefore, future studies must evaluate the potential association between failure of the initial treatment with TMP/SMX and increased mortality in HIV-negative patients. However, it might be difficult to distinguish the reasons for clinical deterioration, including progressive disease, adverse or failed treatment effects, or concomitant infection. In this study, all of our patients were initially treated with TMP/SMZ. Failure of the initial antimicrobial treatment for PCP was significantly associated with increased mortality. After adjusting for potentially confounding factors, failure of the initial antimicrobial treatment was still significantly associated with increased mortality. In most cases of treatment failure, however, the most likely cause of mortality was disease severity (before diagnosis and treatment), rather than drug resistance [33-35]. Regardless, future studies are needed to substantiate our findings with more patients.

There are several potential limitations to our study that should be acknowledged. First, given its retrospective observational design, this study may have been subject to selection bias. In addition, this study was conducted at a single center with a specialized ICU for a large number of cancer patients receiving chemotherapy. Therefore, our findings may not be readily generalizable to other institutions or patient populations. Finally, we could not compare the results with HIV-positive patients, since the number of HIV-positive patients diagnosed with PCP was too small to compare during the 
Table 4 Univariate comparisons of the clinical characteristics of hospital survivors and non-survivors

\begin{tabular}{|c|c|c|c|}
\hline Characteristics & Survivors $(n=28)$ & Non-survivors $(n=23)$ & $P$ value \\
\hline Age, years & $48.0(34.5-59.0)$ & $64.0(50.5-69.0)$ & 0.007 \\
\hline Sex, male & $17(60.7)$ & $18(78.3)$ & 0.298 \\
\hline Underlying disease & & & 0.589 \\
\hline Malignancy & $13(46.4)$ & $15(65.2)$ & \\
\hline Solid organ transplant & $12(42.9)$ & $6(26.1)$ & \\
\hline Others & $3(10.7)$ & $2(8.7)$ & \\
\hline Immunosuppressive agent use, previous month & & & 0.548 \\
\hline Steroid only & $9(32.1)$ & $9(39.1)$ & \\
\hline Chemotherapy only & $4(14.3)$ & $1(4.3)$ & \\
\hline Steroid with chemotherapy & $15(53.6)$ & $13(56.5)$ & \\
\hline Pneumocystis prophylaxis & $0(0.0)$ & $2(8.7)$ & 0.386 \\
\hline \multicolumn{4}{|l|}{ Chest radiography findings } \\
\hline Pleural effusion & $8(28.6)$ & $3(13.0)$ & 0.305 \\
\hline Radiographic pulmonary patterns & & & 1.000 \\
\hline Focal or diffuse alveolar pattern & $13(46.4)$ & $11(47.8)$ & \\
\hline Focal or diffuse interstitial pattern & $3(10.7)$ & $2(8.7)$ & \\
\hline Focal or diffuse alveolar-interstitial pattern & $12(42.9)$ & $10(43.5)$ & \\
\hline Laboratory findings on ICU admission & $9215.0(5320.0-12,225.0)$ & $6980.0(5245.0-12,535.0)$ & 0.757 \\
\hline WBC blood cells, $/ \mu \mathrm{L}$ & $5591.0(2880.0-10,395.0)$ & $5458.0(4235.0-10,960.0)$ & 0.670 \\
\hline Neutrophils, / $\mu \mathrm{L}$ & $648.0(389.5-1278.0)$ & $560.0(278.0-724.5)$ & 0.244 \\
\hline Lymphocytes, / $\mu \mathrm{L}$ & $8.7(4.0-16.0)$ & $11.9(5.1-28.8)$ & 0.232 \\
\hline Albumin, $\mathrm{g} / \mathrm{dL}$ & $2.9(2.5-3.6)$ & $2.8(2.4-3.0)$ & 0.136 \\
\hline $\mathrm{CRP}, \mathrm{mg} / \mathrm{dL}$ & $11.8(8.4-19.1)$ & $15.0(7.4-21.8)$ & 0.570 \\
\hline $\mathrm{PaO}_{2} / \mathrm{FiO}_{2}$ ratio, $\mathrm{mmHg}$ & $147.0(102.3-170.5)$ & $131.2(116.8-160.6)$ & 0.619 \\
\hline $\mathrm{D}(\mathrm{A}-\mathrm{a}) \mathrm{O}_{2}, \mathrm{mmHg}$ & $38.5(30.6-51.5)$ & $41.6(31.1-54.0)$ & 0.771 \\
\hline \multicolumn{4}{|l|}{ Organ failure on ICU admission } \\
\hline Shock & $7(25.0)$ & $5(21.7)$ & 1.000 \\
\hline Renal failure requiring renal replacement therapy & $0(0.0)$ & $1(4.3)$ & 0.921 \\
\hline Respiratory support on ICU admission day & & & 0.016 \\
\hline Mechanical ventilation & $20(71.4)$ & $23(100.0)$ & \\
\hline High-flow nasal cannula & $8(28.6)$ & $0(0.0)$ & \\
\hline \multicolumn{4}{|l|}{ Severity of illness } \\
\hline SAPS3 & $43.0(30.5-54.5)$ & $56.0(46.5-58.0)$ & 0.030 \\
\hline SOFA & $6.0(4.5-7.5)$ & $6.0(5.0-8.5)$ & 0.445 \\
\hline \multicolumn{4}{|l|}{ Other pathogens identified from respiratory specimens } \\
\hline Cytomegalovirus & $3(10.7)$ & $10(43.5)$ & 0.011 \\
\hline Virus other than cytomegalovirus & $4(14.3)$ & $3(13.0)$ & 1.000 \\
\hline Bacteria & $3(10.7)$ & $7(30.4)$ & 0.078 \\
\hline Time to anti-PCP treatment, hours & $64.5(32.5-103.3)$ & $46.2(26.6-91.7)$ & 0.447 \\
\hline Empiric treatment & $16(57.1)$ & $15(65.2)$ & 0.765 \\
\hline Failure to initial treatment & $6(21.4)$ & $16(69.6)$ & 0.002 \\
\hline
\end{tabular}

ICU intensive care unit, $W B C$ white blood cell, $C R P$ C-reactive protein, $\mathrm{PaO}_{2} / \mathrm{FiO}_{2}$ arterial partial pressure of oxygen/fraction of inspired oxygen, $D(A-a) \mathrm{O}_{2}$ alveolararterial oxygen gradient, SAPS 3 Simplified Acute Physiology Score 3, SOFA Sequential Organ Failure Assessment, PCP Pneumocystis pneumonia 
Table 5 Clinical factors affecting hospital mortality

\begin{tabular}{|c|c|c|c|c|c|c|}
\hline & $\underline{\text { Univariable }}$ & & & Multivariable $^{\dagger}$ & & \\
\hline & Crude OR & $95 \% \mathrm{Cl}$ & $P$ value & Adjusted OR & $95 \% \mathrm{Cl}$ & $P$ value \\
\hline Age & 1.06 & $1.01-1.10$ & 0.011 & 1.07 & $1.01-1.14$ & 0.034 \\
\hline Gender & 0.43 & $0.12-1.50$ & 0.184 & 0.80 & $0.10-6.06$ & 0.826 \\
\hline SAPS 3 & 1.05 & $1.00-1.10$ & 0.033 & 1.05 & $0.98-1.13$ & 0.149 \\
\hline Serum albumin, g/dL & 0.47 & $0.18-1.26$ & 0.133 & 0.58 & $0.13-2.56$ & 0.469 \\
\hline Cytomegalovirus co-infection & 5.50 & 1.44-20.96 & 0.013 & 3.19 & $0.52-19.63$ & 0.212 \\
\hline Bacterial co-infection & 3.65 & $0.82-16.19$ & 0.089 & 2.90 & $0.34-24.32$ & 0.327 \\
\hline Time to anti-PCP treatment, hour & 1.00 & $0.99-1.01$ & 0.939 & 1.01 & $0.99-1.02$ & 0.307 \\
\hline Failure to initial treatment & 8.38 & $2.36-29.74$ & 0.001 & 14.12 & $2.23-89.38$ & 0.005 \\
\hline
\end{tabular}

$O R$ odds ratio, $C l$ confidence interval, SAPS Simplified Acute Physiology Score 3, PCP Pneumocystis pneumonia

study period. However, patients with hematologic malignancies and solid tumors receiving chemotherapy do have a higher risk of PCP compared to that of other HIV-negative patients [6-8]. Therefore, our results represent actual practice in the treatment of PCP in HIVnegative patients.

\section{Conclusion}

In conclusion, our data suggest that there is no relationship between the time to anti-PCP treatment and the treatment outcome in $\mathrm{HIV}$-negative patients with PCP and associated hypoxic respiratory failure.

\section{Supplementary information}

Supplementary information accompanies this paper at https://doi.org/10. 1186/s12931-019-1188-6.

Additional file 1. Table S1. Comparison of baseline characteristics of 51 patients according to the quartiles of time to anti-PCP treatment.

Additional file 2. Table S2. Comparison of baseline patient characteristics according to initiation time of anti-PCP treatment.

Additional file 3. Table S3. Comparison of diagnosis and treatment of PCP and clinical outcomes according to initiation time of anti-PCP treatment.

\section{Abbreviations}

BAL: Bronchoalveolar lavage; Cl: Confidence interval; CMV: Cytomegalovirus; $\mathrm{D}(\mathrm{A}-\mathrm{a}) \mathrm{O}_{2}$ : Alveolar-arterial oxygen gradient; DNA: Deoxyribonucleic acid; ED: Emergency department; HIV: Human immunodeficiency virus; ICU: Intensive care unit; IQR: Interquartile range; OR: Odds ratio; PCP: Pneumocystis pneumonia; PCR: Polymerase chain reaction; PF: Arterial partial pressure of oxygen/fraction of inspired oxygen; SAPS 3: Simplified Acute Physiology Score 3; SOFA: Sequential Organ Failure Assessment; TBLB: Transbronchial lung biopsy; TMP/SMX: Trimethoprim/sulfamethoxazole

\section{Acknowledgements}

Not applicable.

\section{Authors' contributions}

REK conceived and designed the study, analyzed the data and drafted this manuscript. SJN, KH and GYS contributed to the design of this study, analysis of the data, and writing of the manuscript. KJ conceived and designed the study, analyzed the data, and wrote the final manuscript. All authors have read and approved the final manuscript.
Funding

This work was supported by a Samsung Medical Center grant (OTA1802901).

\section{Availability of data and materials}

The data that support the findings of this study are available on request from the corresponding author. The data are not publicly available due to privacy or ethical restrictions.

Ethics approval and consent to participate

The Institutional Review Board of Samsung Medical Center approved this study and waived the requirement for informed consent given its

observational nature.

\section{Consent for publication}

Not applicable.

\section{Competing interests}

The authors declare that they have no competing interests.

\section{Author details}

${ }^{1}$ Department of Critical Care Medicine, Samsung Medical Center, Sungkyunkwan University School of Medicine, 81 Irwon-ro, Gangnam-gu, Seoul 06351, Republic of Korea. ²Division of Infectious Diseases, Department of Medicine, Samsung Medical Center, Sungkyunkwan University School of Medicine, Seoul, Republic of Korea. ${ }^{3}$ Division of Pulmonary and Critical Care Medicine, Department of Medicine, Samsung Medical Center, Sungkyunkwan University School of Medicine, 81 Irwon-ro, Gangnam-gu, Seoul 06351, Republic of Korea.

Received: 5 August 2019 Accepted: 11 September 2019

Published online: 26 September 2019

\section{References}

1. Kovacs JA, Hiemenz JW, Macher AM, Stover D, Murray HW, Shelhamer J, et al. Pneumocystis carinii pneumonia: a comparison between patients with the acquired immunodeficiency syndrome and patients with other immunodeficiencies. Ann Intern Med. 1984;100:663-71.

2. Thomas CF Jr, Limper AH. Pneumocystis pneumonia. N Engl J Med. 2004;350:2487-98

3. Bitar D, Lortholary $O$, Le Strat $Y$, Nicolau J, Coignard B, Tattevin P, et al. Population-based analysis of invasive fungal infections, France, 2001-2010. Emerg Infect Dis. 2014;20:1149-55.

4. Kaplan JE, Hanson D, Dworkin MS, Frederick T, Bertolli J, Lindegren ML, et al. Epidemiology of human immunodeficiency virus-associated opportunistic infections in the United States in the era of highly active antiretroviral therapy. Clin Infect Dis. 2000;30(Suppl 1):S5-14.

5. Palella FJ Jr, Delaney KM, Moorman AC, Loveless MO, Fuhrer J, Satten GA, et al. Declining morbidity and mortality among patients with advanced human immunodeficiency virus infection. HIV outpatient study investigators. N Engl J Med. 1998;338:853-60. 
6. Sepkowitz KA, Brown AE, Telzak EE, Gottlieb S, Armstrong D. Pneumocystis carinii pneumonia among patients without AIDS at a cancer hospital. JAMA 1992;267:832-7.

7. Sepkowitz KA. Opportunistic infections in patients with and patients without acquired immunodeficiency syndrome. Clin Infect Dis. 2002;34:1098-107.

8. Zahar JR, Robin M, Azoulay E, Fieux F, Nitenberg G, Schlemmer B. Pneumocystis carinii pneumonia in critically ill patients with malignancy: a descriptive study. Clin Infect Dis. 2002;35:929-34.

9. Salzer HJF, Schafer G, Hoenigl M, Gunther G, Hoffmann C, Kalsdorf B, et al. Clinical, diagnostic, and treatment disparities between HIV-infected and non-HIV-infected immunocompromised patients with Pneumocystis jirovecii pneumonia. Respiration. 2018:96:52-65.

10. Avino LJ, Naylor SM, Roecker AM. Pneumocystis jirovecii pneumonia in the non-HIV-infected population. Ann Pharmacother. 2016;50:673-9.

11. Limper AH, Knox KS, Sarosi GA, Ampel NM, Bennett JE, Catanzaro A, et al. An official American Thoracic Society statement: treatment of fungal infections in adult pulmonary and critical care patients. Am J Respir Crit Care Med. 2011;183:96-128.

12. Bollee G, Sarfati C, Thiery G, Bergeron A, de Miranda S, Menotti J, et al. Clinical picture of Pneumocystis jiroveci pneumonia in cancer patients. Chest. 2007:132:1305-10.

13. Creemers-Schild D, Kroon FP, Kuijper EJ, de Boer MG. Treatment of Pneumocystis pneumonia with intermediate-dose and step-down to lowdose trimethoprim-sulfamethoxazole: lessons from an observational cohort study. Infection. 2016;44:291-9.

14. Kim SJ, Lee J, Cho YJ, Park YS, Lee CH, Yoon HI, et al. Prognostic factors of Pneumocystis jirovecii pneumonia in patients without HIV infection. J Inf Secur. 2014;69:88-95.

15. Liu Y, Su L, Jiang SJ, Qu H. Risk factors for mortality from pneumocystis carinii pneumonia (PCP) in non-HIV patients: a meta-analysis. Oncotarget. 2017:8:59729-39.

16. Roux A, Canet E, Valade S, Gangneux-Robert F, Hamane S, Lafabrie A, et al. Pneumocystis jirovecii pneumonia in patients with or without AIDS, France. Emerg Infect Dis. 2014;20:1490-7.

17. Li MC, Lee NY, Lee CC, Lee HC, Chang CM, Ko WC. Pneumocystis jiroveci pneumonia in immunocompromised patients: delayed diagnosis and poor outcomes in non-HIV-infected individuals. J Microbiol Immunol Infect. 2014:47:42-7.

18. Ko Y, Jeong BH, Park HY, Koh WJ, Suh GY, Chung MP, et al. Outcomes of Pneumocystis pneumonia with respiratory failure in HIV-negative patients. J Crit Care. 2014;29:356-61.

19. Lam WY, Yeung AC, Tang JW, Ip M, Chan EW, Hui M, et al. Rapid multiplex nested PCR for detection of respiratory viruses. J Clin Microbiol. 2007:45:3631-40.

20. Costa C, Delsedime L, Solidoro P, Curtoni A, Bergallo M, Libertucci D, et al. Herpesviruses detection by quantitative real-time polymerase chain reaction in bronchoalveolar lavage and transbronchial biopsy in lung transplant: viral infections and histopathological correlation. Transplant Proc. 2010;42:1270-4.

21. Moreno RP, Metnitz PG, Almeida E, Jordan B, Bauer P, Campos RA, et al. SAPS 3--from evaluation of the patient to evaluation of the intensive care unit. Part 2: development of a prognostic model for hospital mortality at ICU admission. Intensive Care Med. 2005;31:1345-55.

22. Vincent JL, Moreno R, Takala J, Willatts S, De Mendonca A, Bruining H, et al. The SOFA (sepsis-related organ failure assessment) score to describe organ dysfunction/failure. On behalf of the working group on sepsis-related problems of the European Society of Intensive Care Medicine. Intensive Care Med. 1996;22:707-10.

23. Leekha S, Terrell CL, Edson RS. General principles of antimicrobial therapy. Mayo Clin Proc. 2011;86:156-67.

24. Roblot F, Godet C, Le Moal G, Garo B, Faouzi Souala M, Dary M, et al. Analysis of underlying diseases and prognosis factors associated with Pneumocystis carinii pneumonia in immunocompromised HIV-negative patients. Eur J Clin Microbiol Infect Dis. 2002;21:523-31.

25. Walmsley S, Levinton C, Brunton J, Muradali D, Rappaport D, Bast M, et al. A multicenter randomized double-blind placebo-controlled trial of adjunctive corticosteroids in the treatment of Pneumocystis carinii pneumonia complicating the acquired immune deficiency syndrome. J Acquir Immune Defic Syndr Hum Retrovirol. 1995;8:348-57.

26. Smego RA Jr, Nagar S, Maloba B, Popara M. A meta-analysis of salvage therapy for Pneumocystis carinii pneumonia. Arch Intern Med. 2001;161:1529-33.
27. Saah AJ, Hoover DR, Peng Y, Phair JP, Visscher B, Kingsley LA, et al. Predictors for failure of Pneumocystis carinii pneumonia prophylaxis. Multicenter AIDS cohort study. JAMA. 1995;273:1197-202.

28. Bewick V, Cheek L, Ball J. Statistics review 8: qualitative data - tests of association. Crit Care. 2004;8:46-53.

29. Mickey RM, Greenland S. The impact of confounder selection criteria on effect estimation. Am J Epidemiol. 1989;129:125-37.

30. Yoo H, Suh GY, Jeong BH, Lim SY, Chung MP, Kwon OJ, et al. Etiologies, diagnostic strategies, and outcomes of diffuse pulmonary infiltrates causing acute respiratory failure in cancer patients: a retrospective observational study. Crit Care. 2013;17:R150.

31. Azoulay E, Mokart D, Lambert J, Lemiale V, Rabbat A, Kouatchet A, et al. Diagnostic strategy for hematology and oncology patients with acute respiratory failure: randomized controlled trial. Am J Respir Crit Care Med. 2010;182:1038-46.

32. Simonetti A, Viasus D, Garcia-Vidal C, Adamuz J, Roset A, Manresa F, et al. Timing of antibiotic administration and outcomes of hospitalized patients with community-acquired and healthcare-associated pneumonia. Clin Microbiol Infect. 2012;18:1149-55.

33. Festic E, Gajic O, Limper AH, Aksamit TR. Acute respiratory failure due to pneumocystis pneumonia in patients without human immunodeficiency virus infection: outcome and associated features. Chest. 2005:128:573-9.

34. Monnet X, Vidal-Petiot E, Osman D, Hamzaoui O, Durrbach A, Goujard C, et al. Critical care management and outcome of severe Pneumocystis pneumonia in patients with and without HIV infection. Crit Care. 2008;12:R28.

35. Boonsarngsuk V, Sirilak S, Kiatboonsri S. Acute respiratory failure due to Pneumocystis pneumonia: outcome and prognostic factors. Int J Infect Dis. 2009;13:59-66.

\section{Publisher's Note}

Springer Nature remains neutral with regard to jurisdictional claims in published maps and institutional affiliations.

Ready to submit your research? Choose BMC and benefit from:

- fast, convenient online submission

- thorough peer review by experienced researchers in your field

- rapid publication on acceptance

- support for research data, including large and complex data types

- gold Open Access which fosters wider collaboration and increased citations

- maximum visibility for your research: over $100 \mathrm{M}$ website views per year

At $\mathrm{BMC}$, research is always in progress.

Learn more biomedcentral.com/submissions 